\title{
Interação pai-criança e problemas externalizantes na infância
}

\author{
Luna Maiana Araújo Freitas \\ Patrícia Alvarenga \\ Universidade Federal da Bahia, BA, Brasil
}

\begin{abstract}
Resumo
O presente estudo avaliou a relação entre as habilidades sociais e práticas educativas negativas paternas e os problemas externalizantes na infância. Participaram do estudo 33 pais de crianças entre seis e nove anos. Os pais responderam ao Roteiro de Entrevista das Habilidades Sociais Educativas Parentais (RE-HSE-P) e ao Inventário de Comportamentos da Infância e Adolescência 6-18 anos (CBCL). Os resultados revelaram correlações positivas entre as práticas negativas paternas e os problemas externalizantes. Discutem-se as implicações das práticas negativas para o desenvolvimento de problemas externalizantes. Destaca-se a importância de considerar o papel mediador da interação mãe-filho sobre as relações entre as práticas paternas e estes problemas.
\end{abstract}

Palavras-chave: Problemas externalizantes; Habilidades sociais; Relações pai-criança.

\section{Father-child interaction and externalizing behaviors in childhood}

\begin{abstract}
This study evaluated the relation between the paternal social childrearing skills and the externalizing behaviors in childhood. Thirty-three fathers of children aged between six and nine years participated in this study. The fathers responded to the Interview Script of Parental Social Childrearing Skills (RE-HSE-P) and the Child Behavior Checklist 6-18 years (CBCL). The results revealed positive correlations between negative paternal practices and externalizing behaviors. The implications of negative practices for the development of externalizing behaviors are discussed. The importance of considering the mediating role of mother-child interaction on the relations between paternal practices and externalizing behaviors were also highlighted.
\end{abstract}

Keywords: Externalizing problems; Social skills; Father child relations.

\section{Interacción padre-hijo y externalización de problemas en la infancia}

\section{Resumen}

El presente estudio evaluó la relación entre las habilidades sociales y las prácticas negativas paternas con los problemas de comportamento externalizantes en la infancia. El estúdio incluyó a 33 padres de niños entre los seis y nueve años. Los padres respondieron al Roteiro de Entrevista sobre Habilidades Sociales Educativas Paternas (RE-HSE-P) y al Inventario de Comportamientos de la Infancia y Adolescencia 6-18 años (CBCL). Los resultados revelaron correlaciones positivas entre las prácticas negativas y los problemas externalizantes. Se discuten las implicaciones de las prácticas negativas para el desarrollo de los problemas externalizantes. Se destaca la importancia de considerar el papel mediador de la interacción madre-hijo, sobre las relaciones entre las prácticas paternas y estos problemas.

Palavras-chave: Externalización de problemas; Habilidades sociales; Relaciones padre-niños. 
Desfechos desenvolvimentais negativos ou indesejados e repertórios comportamentais atípicos têm suscitado o interesse de muitos pesquisadores (Shaw, Bell, \& Gilliom, 2000). Nesse contexto, desde a década de 1960, o conceito de problemas de comportamento tem sido utilizado (Achenbach, 1966) para fazer referência a déficits ou excessos comportamentais que produzem prejuízos para a interação da criança com as outras pessoas e limitam seu acesso a contingências de reforçamento (Bornstein, Hahn, \& Haynes, 2010). Dentre os problemas de comportamento, os problemas externalizantes compreendem um conjunto de queixas como agressividade, impulsividade, desobediência, oposicionismo, temperamento exaltado, roubos ou fugas (Achenbach \& Rescorla, 2001).

Dados sobre prevalência, tanto de estudos brasileiros quanto internacionais, indicam valores que variam, aproximadamente, entre $20 \%$ e $25 \%$ para os problemas externalizantes (Sherman, Duarte, \& Verdeli, 2011). Considerando-se as evidências empíricas acerca da relação entre os problemas de comportamento no início do desenvolvimento e a ocorrência de psicopatologias no futuro (Achenbach, 1991; APA, 2002), tais indicadores sinalizam a relevância de investigar fatores associados ao desenvolvimento dos problemas de comportamento na infância, tendo em vista a relevância de propor intervenções precoces, de forma a minimizar prejuízos ao longo do desenvolvimento (Trautmann-Villalba, Gschwendt, Schmidt, \& Laucht, 2006).

Evidências empíricas acerca dos preditores e variáveis relacionadas à aquisição e manutenção dos problemas externalizantes apontam para fatores relacionados, por um lado, aos mecanismos biológicos e à hereditariedade (Gracioli \& Linhares, 2014) e, por outro, aos fatores socioambientais (Rohenkohl $\&$ Castro, 2012). Neste segundo grupo de variáveis, destacam-se as características e a dinâmica das relações da família, principalmente, as interações entre pais e filhos. Nesse sentido, a forma como os pais interagem e as estratégias que utilizam para socializar e cuidar de seus filhos influenciam o desenvolvimento da criança (Grusec, 2011), podendo atuar como fatores de risco ou de proteção (Bolsoni-Silva \& Marturano, 2010).

Del-Prette e Del-Prette (2008) e Bolsoni-Silva, Del-Prette e Oishi (2003), apresentaram um modelo teórico para a compreensão e avaliação da interação entre pais e filhos, baseados no trabalho inicial de Argyle (1980 in Del-Prette \& Del-Prette, 2008) sobre a tarefa educativa formal de professores. Os autores descreveram o conceito de Habilidades Sociais Educativas Parentais (HSE-P), que seriam respostas usadas para a promoção do desenvolvimento e da aprendizagem dos filhos (Bolsoni-Silva \& Loureiro, 2010; Del-Prette \& Del-Prette, 2008). As HSE-P se agrupam em três dimensões. A categoria comunicação é composta pelos comportamentos verbais de pais e cuidadores que incluem iniciar e manter conversa, fazer perguntas e ouvir atentamente perguntas feitas pelos filhos. A categoria expressão de sentimentos e enfrentamento compreende os comportamentos de pais e cuidadores de forma a expressar sentimentos positivos, negativos e opiniões, as demonstrações de carinhos e as interações que envolvem brincadeiras. Por fim, a categoria estabelecimento de limites inclui os comportamentos de pais ou cuidadores de identificar comportamentos adequados e inadequados das crianças, cumprir promessas, concordância com o cônjuge e identificar os próprios "erros".

Embora poucos estudos adotem o modelo das HSE-P, a literatura já acumula importantes evidências sobre as relações entre características da interação pai-criança e os problemas externalizantes. Práticas e interações pai-criança que são contempladas no modelo das HSE-P, a saber, comunicação, expressão se sentimentos e estabelecimentos de regras, tem sido investigadas com relação ao comportamento infantil. Muitos desses estudos destacam, por um lado, o impacto das habilidades paternas, e por outro, a repercussão de práticas de caráter negativo ou coercitivo, de modo semelhante ao que o modelo das HSE-P propõe.

Raya, Pino e Herruzo (2011) sugeriram que as habilidades de comunicação do pai com o filho parecem funcionar como um preditor das habilidades sociais da criança e, ao mesmo tempo, como um fator de proteção contra o desenvolvimento de problemas externalizantes. Outra habilidade paterna que parece estar associada a menor frequência de problemas externalizantes e repertório mais amplo de habilidades sociais da criança é a expressão de sentimentos e estratégias de enfrentamento dos pais. Um estudo indicou que, quanto mais expressões de afeto positivo e quanto maior a tendência do pai a resolver conflitos utilizando o estilo autoritativo (quando o pai interage com o filho de forma exigente e responsiva, por exemplo, estabelecendo regras e incentivando o diálogo, sendo amoroso e envolvido ao mesmo tempo), menores os escores de problemas externalizantes e mais socialmente habilidosos são os filhos (Harper, 2010).

Da mesma forma, as práticas disciplinares e as habilidades paternas de estabelecer limites têm sido descritas na literatura como um importante preditor do desenvolvimento socioemocional da criança. Nesse sentido, algumas estratégias utilizadas por pais e mães 
parecem fundamentais para promover a competência social e inibir os problemas externalizantes, como o uso de contingências de reforçamento de forma eficaz (Patterson, Reid, \& Dishion, 1992; Patterson, 2002). Ao mesmo tempo, a literatura sugere que quanto mais autoritários e inconsistentes os pais e quanto mais práticas coercitivas, controle psicológico e conflito com os filhos, maiores os escores de problemas externalizantes das crianças (Harper, 2010; Marin, Piccinini, Gonçalves, \& Tudge, 2012; Zhang, 2013).

Os preditores do desenvolvimento infantil relacionados à paternidade e à interação paicriança ainda não foram amplamente investigados, especialmente no contexto brasileiro, em que os pesquisadores têm se voltado predominantemente para tipos de práticas e características maternas ou para a comparação entre práticas maternas e paternas na interação com seus filhos (Leme \& Marturano, 2014; Marin et al., 2012; Bolsoni-Silva \& Marturano, 2010). Embora a literatura acumule evidências sobre o papel do pai no desenvolvimento e na melhoria de dificuldades comportamentais e emocionais da criança, poucos são os estudos brasileiros que investigam essas relações (Bueno \& Vieira, 2014).

Pesquisas que tenham como objeto de estudo a paternidade e sua relação com o desenvolvimento infantil se fazem necessárias para um melhor entendimento das diferenças no comportamento de pais e de mães e sobre o impacto particular que cada um deles produz no desenvolvimento de seus filhos. Nesse contexto, se insere o presente estudo que teve como objetivo avaliar a relação entre o repertório de habilidades sociais e práticas educativas negativas paternas e os problemas externalizantes em crianças de seis a nove anos. As hipóteses investigadas foram as de que quanto maiores os escores de habilidades sociais educativas paternas menores os escores de problemas externalizantes dos filhos, e de que quanto maiores os escores de práticas educativas negativas dos pais, maiores os escores de problemas externalizantes dos filhos.

\section{Método}

\section{Participantes}

Participaram deste estudo 33 pais de crianças de ambos os sexos, com idade entre seis e nove anos, residentes na cidade de Feira de Santana-BA, selecionados por acessibilidade em escolas públicas e privadas da rede de ensino do município. Os critérios para a inclusão de participantes na amostra foram os seguintes: (a) ter idade igual ou superior a 19 anos; (b) residir com a criança alvo do estudo; (c) residir com a mãe biológica da criança, seja casado ou em união estável; e, (d) ser pai biológico da criança. Os critérios de inclusão foram estabelecidos na tentativa de produzir uma amostra mais homogênea. A opção por incluir pais biológicos que residissem com as crianças visou a inclusão de pais que convivessem com a criança e que pudessem ser bons informantes, tanto dos comportamentos de seus filhos, quanto de seus próprios comportamentos na interação com a criança.

A idade média dos pais foi de 38,82 anos $(\mathrm{DP}=6,93)$ e a escolaridade média foi de 11,91 anos $(\mathrm{DP}=2,81)$. $\mathrm{O}$ número médio de filhos dos pais participantes foi de 2,33 ( $\mathrm{DP}=0,92)$. A média da renda familiar foi de $\mathrm{R} \$ 4.480,24(\mathrm{DP}=2.650,44)$, com mediana de $\mathrm{R} \$ 4.000,00(\mathrm{Md}=4.000)$. Os filhos tinham idade média de 7,76 $(\mathrm{DP}=1,14)$, sendo que 14 eram meninos $(42,4 \%)$ e 19 eram meninas (57,6\%). Na amostra, 22 crianças frequentavam escola pública $(66,7 \%)$ e $11(33,3 \%)$ frequentavam escolas privadas. O tipo de relação dos participantes com a mãe da criança variou entre as seguintes condições: 27 (81,8\%) eram casados e seis $(18,2 \%)$ viviam em união estável, sendo a média do tempo de coabitação de 13,42 anos $(\mathrm{DP}=5,30)$.

\section{Delineamento e Procedimento}

O estudo adotou um delineamento correlacional. Inicialmente, o projeto de pesquisa foi submetido e aprovado pelo Comitê de Ética em Pesquisa da Escola de Enfermagem da Universidade Federal da Bahia no parecer de número 832.942. Em seguida, foram realizados contatos com instituições de educação infantil, convidando os pais das crianças matriculadas a participarem da pesquisa. Através de um contato telefônico prévio, autorizado pelos pais, foi marcada uma visita domiciliar ou à escola para a coleta de dados, durante a qual os participantes assinaram o Termo de Consentimento Livre e Esclarecido, e responderam à Ficha de Dados Sociodemográficos, ao Inventário de Comportamentos da Infância e Adolescência (CBCL) e ao Roteiro de Entrevista de Habilidades Sociais Educativas Parentais (RE-HSE-P). Todos os instrumentos foram aplicados na forma de entrevista, isto é, a leitura dos itens e o registro das respostas foram feitos pelo pesquisador. Cada sessão de aplicação dos instrumentos teve duração de aproximadamente uma hora e foi gravada em áudio.

\section{Instrumentos}

Ficha de dados sociodemográficos: preenchida pelo pesquisador com informações fornecidas pelos pais. Teve por objetivo investigar dados demográficos da família, como a idade, a escolaridade, a profissão 
e o estado civil dos pais, a existência de outros filhos, a renda familiar, o número de pessoas que residem na casa, dentre outras informações.

Roteiro de Entrevista de Habilidades Sociais Educativas Parentais - RE-HSE-P (Bolsoni-Silva, Loureiro \& Marturano, 2011): consiste numa entrevista semiestruturada, que tem como objetivo descrever funcionalmente a capacidade social e educacional relacionadas à interação entre pais e filhos. A aplicação é individual, sem limite de tempo, através de 13 perguntas guia, cujas respostas são posteriormente codificadas conforme o manual do instrumento e incluídas em cinco categorias. Para o presente estudo foram utilizadas duas delas. A primeira, das habilidades sociais educativas paternas (HSE-P) inclui as práticas educativas parentais positivas, associadas ao favorecimento do desenvolvimento infantil como, por exemplo, quando o pai interage com o filho elogiando, incentivando, falando/conversando, ou expressandose na ausência do filho. A segunda, categoria das práticas educativas negativas (PR-NEG), que compõe aspectos negativos da interação, mensura respostas paternas como dizer não sem explicação, ameaçar, retirar privilégios. São produzidos escores para cada categoria, compostos a partir do número de itens citados pelos pais, que são categorizados em clínico, limítrofe e não clínico. Para as habilidades sociais educativas a categoria não clínica envolve escores a partir de 10 e a classificação clínica (que no presente estudo inclui também escores limítrofes), escores entre zero e nove. Para as práticas negativas, os escores da categoria não clínica variam entre zero e cinco e clínica a partir de seis. Um estudo sobre as propriedades psicométricas do instrumento (Bolsoni-Silva \& Loureiro, 2010) apontou valores satisfatórios de consistência interna (alfa de Cronbach de 0,84).

Inventário de Comportamentos da Infância e Adolescência 6-18 anos - Child Behavior Checklist (CBCL): O inventário é uma versão brasileira do Child Behavior Checklist (CBCL) que tem como objetivo medir os problemas de comportamento em criança e adolescentes, de 6 a 18 anos, de acordo com as informações fornecidas pelos seus pais ou cuidadores (Achenbach \& Rescorla, 2001; Bordin et al., 2013). O cuidador da criança deve indicar a frequência com que esses problemas ocorrem, em uma escala de zero a dois. O instrumento é dividido em 11 escalas e composto por 138 itens. Para o presente estudo foi utilizada apenas a escala de problemas de comportamento externalizantes, formada pelas síndromes comportamento de quebrar regras e comportamento agressivo, e as escalas baseadas no Manual de Diagnóstico e Estatística de Transtornos Mentais (DSM-IV-TR), problemas de oposição e desafio e problemas de conduta. Em todas as escalas do CBCL/6-18, o escore é classificado, conforme propõe o instrumento, como "clínico", "limítrofe" ou "não clínico", de acordo com a amostra normativa de pares (Achenbach \& Rescorla, 2001; Bordin, et al., 2013). As categorias limítrofes e clínica indicam alta probabilidade de que a criança ou adolescente avaliado desenvolva transtornos mentais associados aos sintomas incluídos na escala de problemas externalizantes, por isso, no presente estudo, os escores classificados como limítrofes também compuseram a categoria clínica. Para a escala de problemas de comportamento externalizante, os escores menores que 60 compõem a categoria não clínica e a partir de 60 a categoria clínica. Para as síndromes comportamento de quebrar regras e comportamento agressivo, e as escalas baseadas no DSM-IV-TR, problemas de oposição e desafio e problemas de conduta, os pontos de corte são os mesmos, até 65 não clínica, e maior que 69 , clínica.

\section{Resultados}

Inicialmente foram analisados os escores de problemas externalizantes da amostra e os escores das síndromes e escalas relacionadas a essas queixas. $\mathrm{Na}$ escala de problemas de comportamento externalizantes a média dos escores foi de $\mathrm{M}=54,09 \quad(\mathrm{DP}=7,96)$. Nas síndromes que compõem a escala de problemas externalizantes, os escores médios obtidos foram de $\mathrm{M}=53,30(\mathrm{DP}=4,18)$ em comportamento de quebrar regras e de $\mathrm{M}=56,88(\mathrm{DP}=6,81)$ na síndrome de comportamento agressivo. Nas escalas baseadas no Manual de Diagnóstico e Estatística de Transtornos Mentais (DSM-IV-TR), a média dos escores de problemas de oposição e desafio foi de $M=55,21$ $(\mathrm{DP}=7,03)$ e em problemas de conduta o escore médio foi de $\mathrm{M}=53,15(\mathrm{DP}=3,36)$. Os escores médios, tanto das escalas como das síndromes, situam-se abaixo dos pontos de corte que determinam as categorias limítrofe e clínica.

Na segunda etapa de análise foram considerados os escores de habilidades sociais educativas e de práticas educativas negativas da amostra. A média dos escores das habilidades sociais educativas paternas para a amostra total foi de $\mathrm{M}=7,85(\mathrm{DP}=2,38)$, indicando déficit nas habilidades sociais educativas. Conforme os escores, os participantes foram divididos nas categorias não clínica e limítrofe-clínica. A minoria dos pais $(\mathrm{n}=7)$ obteve escores classificados como não clínicos. Nesse grupo o escore médio de habilidades sociais educativas paternas foi de $\mathrm{M}=11,57$ ( $\mathrm{DP}=1,51)$. A maioria dos pais $(n=26)$ que compuseram a amostra ficou na 
categoria clínica, com escores médios de $\mathrm{M}=6,85$ $(\mathrm{DP}=1,84)$. Para as práticas educativas negativas a média da amostra total foi de $\mathrm{M}=4,82(\mathrm{DP}=3,58)$ que se classifica na categoria não clínica (menores que cinco), indicando que os pais da amostra relataram poucos episódios de interação negativa. Quando os participantes foram divididos nas categorias não clínica e limítrofe-clínica, 20 pais ficaram na categoria não clínica, apresentando média de $\mathrm{M}=2,50(\mathrm{DP}=1,60)$. Os 13 pais classificados na categoria clínica obtiveram escores médios de $\mathrm{M}=8,38(\mathrm{DP}=2,72)$, indicando o uso expressivo de práticas como ameaçar, bater, gritar, retirar privilégios e dizer não sem explicação.

Por fim, foram analisadas as correlações entre as habilidades sociais educativas e práticas negativas paternas e os problemas externalizantes. A Tabela 1 mostra os resultados do teste de correlação de Spearman.

\section{TABELA 1}

Correlações entre os escores das escalas de problemas externalizantes das crianças e das categorias de habilidades sociais educativas e práticas educativas negativas dos pais $(n=33)$

\begin{tabular}{lcc}
\hline \multicolumn{1}{c}{ Escalas do CBCL } & $H S E-P$ & PR-NEG \\
\hline $\begin{array}{l}\text { Problemas de Comportamento } \\
\text { Externalizantes }\end{array}$ & 0,20 & $0,34^{*}$ \\
Comportamento de Quebrar Regras & 0,17 & $0,53^{* *}$ \\
Comportamento Agressivo & 0,16 & 0,27 \\
Escalas baseadas no DSM-IV-R & & \\
Problemas de Oposição e Desafio & 0,11 & 0,26 \\
Problemas de Conduta & 0,00 & $0,51^{* *}$ \\
\hline
\end{tabular}

$* \mathrm{p}<0,05 ; * * \mathrm{p}<0,01$.

Os resultados revelaram ausência de correlações significativas entre os escores dos problemas externalizantes dos filhos e os escores das habilidades sociais educativas paternas. Por outro lado, as análises demonstraram a presença de fraca correlação positiva $(\rho=0,34, p<0,05)$ entre a categoria de práticas educativas negativas e a escala problemas externalizantes, e correlação positiva moderada $(\rho=0,53, p<0,01)$ entre a categoria de práticas educativas negativas e a síndrome de comportamento de quebrar regras do CBCL, bem como entre a mesma categoria e a escala baseada no DSM-IV-TR, problemas de conduta $(\rho=0,51, p<0,01)$. Esses resultados indicam que quanto mais práticas educativas negativas os pais utilizam para lidar com seus filhos, mais problemas externalizantes as crianças tendem a apresentar.

\section{Discussão}

O presente estudo teve por objetivo avaliar a relação entre o repertório de habilidades sociais educativas e de práticas negativas paternas e os problemas externalizantes na infância. A hipótese inicial do estudo era de que quanto maior o repertório de habilidades sociais educativas paternas menores seriam os escores de problemas externalizantes dos filhos. Ao mesmo tempo, esperava-se que quanto mais práticas educativas negativas os pais utilizassem, maiores seriam os escores dos problemas externalizantes das crianças.

A primeira hipótese não foi confirmada. As habilidades sociais educativas dos pais não apresentaram correlação com os escores de problemas externalizantes. Esse achado vai de encontro aos resultados de grande parte das pesquisas anteriores, que sugerem o impacto das habilidades paternas, assim como das maternas, como fator de proteção contra o desenvolvimento de problemas externalizantes, bem como no desenvolvimento de comportamentos prósociais e da competência social da criança (Fantinato \& Cia, 2015; Torres, Verissimo, Monteiro, Ribeiro, \& Santos, 2014).

Entretanto, embora escassos, alguns trabalhos também alcançaram resultados na mesma direção dos achados do presente estudo, a saber, a ausência de efeito do envolvimento e interação positiva do pai no desenvolvimento da criança, como sugeriram Sarkadi, Kristiansson, Oberklaid e Bremberg (2008) e McLoyd, Cauce, Takeuchi e Wilson (2000) em estudos de revisão sistemática. Outros estudos também verificaram a ausência de relações significativas entre práticas educativas e habilidades sociais parentais e problemas externalizantes/agressividade, no contexto da maternidade, como ressaltaram Lins, Alvarenga, Paixão, Almeida e Costa (2012) num estudo de revisão da literatura nacional. Da mesma forma, Pougnet, Serbin, Stack e Schwartzman (2011) também não encontraram relação entre o envolvimento paterno e os problemas externalizantes. Braza et al. (2015) também verificaram resultados semelhantes com famílias espanholas e não encontraram relações entre os problemas externalizantes e os estilos paternos. Torres et al. (2014) acrescentaram, mais especificamente, a respeito dos efeitos das interações e envolvimento do pai sobre os resultados desenvolvimentais das crianças, que estes estão relacionados a uma série de fatores como variáveis do pai, da criança e da família, que precisam ser consideradas. 
Além dos aspectos conceituais e metodológicos que poderiam explicar tais diferenças entre os achados de diferentes estudos, outras duas hipóteses explicativas também são plausíveis. O primeiro aspecto a ser considerado diz respeito à participação dos pais na educação dos filhos. Apesar das mudanças recentes na função social e papel paterno na família e nos cuidados com os filhos (Lamb, 2012), os pais ainda se envolvem e participam dos cuidados e educação dos filhos em menor frequência do que as mães, como sugerem alguns estudos (Gryczkowski, Jordan, \& Mercer, 2010). Gryczkowski et al., (2010) verificaram que as mães relataram níveis mais elevados de envolvimento e práticas positivas, quando comparadas aos pais. Na mesma direção Rabinovich, Moreira e Franco (2012), investigando 170 famílias baianas, constataram que as mães oferecem mais suporte emocional e se envolvem mais em atividades de cuidados e brincadeiras em comparação com os pais. É importante destacar o fato de que a maior parte da amostra no presente estudo obteve escores clínicos em habilidades sociais educativas, o que indica que elas foram relatadas pelos pais com baixas frequências, sugerindo que o repertório de habilidades dos participantes era restrito. Nesse sentido, pode ser que os pais tenham menos oportunidades do que as mães de interagirem com seus filhos em contextos ou situações que permitam a aproximação e interações positivas. Desse modo, as habilidades ou práticas paternas positivas teriam menor probabilidade de afetar o comportamento infantil por serem menos frequentes os episódios de interação em contextos positivos.

A explicação para este resultado pode estar ainda relacionada às reservas comportamentais das crianças, talvez influenciadas pelas práticas positivas e facilitadoras das mães. Nesse sentido, alguns estudos recentes têm investigado a influência simultânea das estratégias ou práticas educativas maternas e paternas para a aquisição e manutenção de certos repertórios de seus filhos e encontraram resultados importantes, sugerindo o papel mediador das práticas maternas no efeito da interação de pais para o desenvolvimento infantil, como os estudos de Murray, Dwyer, Rubin, Knighton-Wisor e Booth-LaForce (2014) e Verhoeven, Junger, van Aken, Deković e van Aken (2010). Esses achados ilustram, como afirmou Jenkins (2012), que um dos desafios mais significativos no estudo da parentalidade é isolar os papéis individuais nas relações parentais, sugerindo que investigações que considerem o contexto familiar em toda sua complexidade constituiriam um mecanismo importante para alcançar esse objetivo.
Os resultados corroboraram a segunda hipótese do estudo, indicando que quanto mais práticas negativas os pais utilizaram, mais problemas externalizantes seus filhos apresentaram. Esses resultados apoiam os achados da literatura, que apontam para os efeitos deletérios das estratégias coercitivas para o desenvolvimento de problemas de comportamento (Harper, 2010; Patterson, 2002; Zhang, 2013).

No presente estudo as práticas paternas negativas avaliadas através do RE-HSE-P envolvem interações do pai com a criança nas quais ele pode ameaçar bater e retirar privilégios, colocar a criança de castigo, bater, gritar, brigar, xingar, dizer não sem explicação, dar bronca de forma autoritária, fazer acusações sobre o cônjuge, fazer chantagem, entre outras possibilidades, que são práticas do tipo não habilidosas ativas. $\mathrm{O}$ instrumento também descreve outras interações paternas como fazer comentários sem entusiasmo, não fazer nada, observar, ouvir e tentar se esquivar, que são consideradas práticas não habilidosas passivas. Outas práticas negativas envolvem o pai sentir-se mal, chateado, frustrado, impotente, preocupado, nervoso, sem autoridade, dentre outras. Os resultados demonstraram que a utilização de práticas como essas está relacionada a comportamentos infantis como mentir, ameaçar pessoas, discutir muito, desobedecer, perturbar os outros, agredir os outros, dentre outros problemas externalizantes. Outros estudos verificaram as mesmas relações (Harper, 2010; Marin et al., 2012; Raya et al., 2011; Trautmann-Villalba et al., 2006).

Esses achados, do presente estudo e dos demais, fortalecem a relação entre os problemas externalizantes das crianças e certos comportamentos paternos que envolvem estratégias coercitivas. A esse respeito, Guilhardi (2002) sugeriu que essas interações podem incluir contingências coercitivas, como, por exemplo, repreender e se afastar, que produzem ao mesmo tempo, tanto comportamentos inadequados de contra controle e oposição como mentir ou agredir, quanto sentimentos desagradáveis como a ansiedade, a raiva e a culpa. Quando utilizam estratégias coercitivas, os pais têm como objetivo interromper comportamentos que consideram inadequados; para tanto lançam mão de estimulação aversiva ameaçando bater ou retirar privilégios, repreendendo verbalmente o comportamento do filho, o colocando de castigo ou suspendendo reforçadores e forçando fisicamente a criança a se comportar de determinada forma, ou ainda batendo na criança. Segundo alguns autores (Patterson, 2002; Sidman, 1989/2003; Todorov, 2001) a manutenção do comportamento de punir está relacionada principalmente aos efeitos da punição 
sob o comportamento do punidor, como a cessação imediata da resposta punida, ou seja, produzindo reforço negativo quando, diante dos berros do pai, a criança interrompe um comportamento indesejado. Ao utilizarem tais formas de controle, os pais podem, em curto prazo eliminar os comportamentos inadequados, entretanto, ao mesmo tempo estão oferecendo modelos e favorecendo a aquisição e manutenção de problemas externalizantes. Além disso, tais estratégias dos pais podem estar modelando comportamentos discriminados a partir de antecedentes que sinalizam a punição, como a presença do pai, porém, mantendo o padrão de comportamentos inadequados em outros contextos e exigindo, para controlar a resposta punida, a presença do agente controlador, no caso, o pai.

Um outro efeito observado é a pouca efetividade dessas práticas para modelar comportamentos adequados, já que ao utilizarem práticas coercitivas os pais não demonstram qual seria o comportamento adequado a ser emitido. Outro efeito importante diz respeito às emoções. As crianças podem desenvolver respostas emocionais associadas ao estímulo antecedente à emissão de comportamentos inadequados. Nesse caso, o próprio pai ou situações relacionadas a ele podem adquirir função aversiva, comprometendo o relacionamento e a aproximação entre pai e filho. O uso da punição também pode estar acompanhado de reforçadores. Especialmente quando a criança está sofrendo privação de afeto e atenção do pai, emitir comportamentos inadequados pode ser a principal fonte de reforçadores, tornando a punição um reforçador condicionado. Ao mesmo tempo, o uso dessas práticas pode desfavorecer o uso de práticas mais contingentes, assertivas e reforçadoras para pais e filhos, que contribuam para um relacionamento mais favorável ao desenvolvimento socioemocional da criança. Sendo assim, os efeitos colaterais da punição acabam por suprimir os possíveis benefícios produzidos por ela, especialmente na falta de modelos ou fontes de reforçamento alternativas (Sidman, 1989/1995), como as práticas maternas positivas facilitadoras.

Vale ressaltar ainda que a utilização tanto de práticas como a punição verbal, ameaça de punição, privação, coação e punição física, quanto de estratégias permissivas e negligentes também podem ser influenciadas pelo contexto cultural. Nesse sentido, como salientou Guilhardi (2002) muitos dos erros dos pais na educação dos filhos ocorrem por falta de conhecimento sobre como, por um lado, minimizar ou eliminar comportamentos inadequados e, por outro, instalar e manter comportamentos adequados, sinalizando a necessidade de estratégias educativas e informativas, possivelmente podendo-se acrescentar o desconhecimento sobre os efeitos deletérios das práticas negativas.

Poucos estudos têm sido hábeis em isolar mecanismos precisos pelos quais tipos específicos de práticas paternas podem ser relacionadas tanto a comportamentos pró-sociais quanto aos problemas de comportamento na infância (Fagan, Day, Lamb, \& Cabrera, 2014). De uma forma geral, parte dessa dificuldade em compreender o papel isolado do pai para o desenvolvimento infantil pode ser justificada pelo caráter dinâmico e relacional dessas interações. O presente estudo compartilha dessa limitação, especialmente devido ao número reduzido de participantes na amostra e à falta de controle de variáveis importantes, como a qualidade das interações maternas e do relacionamento conjugal, para a explicação dos problemas externalizantes. Contudo, ressalta-se o caráter exploratório deste trabalho, especialmente a ênfase sobre a interação pai-criança, que muitas vezes tem papel coadjuvante, mesmo diante das evidências sobre o impacto da relação pai-criança sobre o desenvolvimento (Harper, 2010; Lamb, 2012; Phares et al., 2005; Trautmann-Villalba et al., 2006; Zhang, 2013).

\section{Considerações Finais}

Os achados do presente estudo sugerem que as práticas educativas negativas paternas se associam de forma consistente aos problemas externalizantes, embora o papel desses fatores na aquisição e manutenção desse padrão deva ser considerado com cautela, devido ao caráter correlacional do delineamento adotado. Ao mesmo tempo, a ausência de relações entre as habilidades sociais educativas paternas e os problemas externalizantes aponta para a importância de se considerar também as características da interação mãe-filho e os contextos específicos em que as habilidades paternas ocorrem, indicando a importância de novas investigações que adotem delineamentos mais sofisticados, que permitam a busca de aprofundamento sobre as contingências em que as habilidades e as práticas negativas paternas ocorrem, assim como a possibilidade de analisar os efeitos da qualidade da interação mãe-filho sobre as relações entre as práticas paternas e os problemas externalizantes. O presente estudo também aponta para a importância de esforços para a divulgação, de caráter preventivo, de informações sobre os efeitos deletérios das práticas negativas e ao mesmo tempo a promoção de estratégias de intervenção que visem a favorecer as habilidades sociais educativas dos pais. 


\section{Referências}

Achenbach, T. M. (1966). The classification of children's psychiatric symptoms: a factor-analytic study. Psychological Monographs: General and Applied, 80(7), 1-37. http://dx.doi.org/10.1037/h0093906

Achenbach, T. M. (1991). Manual for The Child Behavior Checklist/4-18 and 1991 Profile. Burlington: University of Vermont Department of Psychiatry.

Achenbach, T. M. \& Rescorla, L. A. (2001). Manual for ASEBA School-Age Forms \& Profiles. Burlington: University of Vermont, Research Center for Children, Youth, \& Families.

American Psychiatric Association (2002). Diagnostic and Statistical Manual of Mental Disorders: DSM-IV-TR (4ํㅡㄹ. ed.). Washington, DC: American Psychiatric Association.

Bolsoni-Silva, A. T. \& Loureiro, S. R. (2010). Validação do Roteiro de Entrevista de Habilidades Sociais Educativas Parentais (RE-HSE-P). Avaliação Psicológica, 9(1), 63-75.

Bolsoni-Silva, A.T., Loureiro, S.R., \& Marturano, E. M. (2011). Roteiro de Entrevista de Habilidades Sociais Parentais - RE-HSE-P. São Paulo: Vetor.

Bolsoni-Silva, A. T., \& Marturano, E. M. (2010). Relacionamento conjugal, problemas de comportamento e habilidades sociais em pré-escolares. Psicologia: Teoria e Pesquisa, 26(1), 67-75. http://dx.doi.org/10.1590/S010237722010000100009

Bolsoni-Silva, A. T., Prette, A. Del, \& Oishi, J. (2003). Habilidades sociais de pais e problemas de comportamento de filhos. Argumento, V(9), 11-30. S1413-03942008000100010

Bordin, I. A., Rocha, M. M., Paula, C. S., Teixeira, M. C. T. V, Achenbach, T. M., Rescorla, L. A., \& Silvares, E. F. M. (2013). Child Behavior Checklist (CBCL), Youth Self-Report (YSR) and Teacher's Report Form (TRF): an overview of the development of the original and Brazilian versions. Cadernos de Saúde Pública, 29(1), 13-28. http://dx.doi. org/10.1590/S0102-311X2013000500004

Bornstein, M. H., Hahn, C. S., \& Haynes, O. M. (2010). Social competence, externalizing, and internalizing behavioral adjustment from early childhood through early adolescence: developmental cascades. Development and Psychopathology, 22(4), 717-35. http://dx.doi.org/10.1017/S0954579410000416

Braza, P., Carreras, R., Mu-oz, J. M., Braza, F., Azurmendi, A., Pascual-Sagastizábal, E., ..., \& Sánchez-Martín, J. R. (2015). Negative Maternal and Paternal Parenting Styles as Predictors of Children's Behavioral Problems: Moderating Effects of the Child's Sex. Journal of Child and Family Studies, 24(4), 847-856. http://dx.doi.org/10.1007/s10826013-9893-0

Bueno, R. K. \& Vieira, M. L. (2014). Análise de estudos brasileiros sobre o pai e o desenvolvimento infantil. Psicologia Argumento, 32(76), 151-159. http://dx.doi.org/10.7213/psicol.argum.32.076.AO10

Del-Prette, Z. A. \& Del-Prette, A. (2008). Um sistema de categorias de habilidades sociais educativas. Paidéia, 18(41), 517-530. http://dx.doi.org/10.1590/S0103-863X2008000300008

Fagan, J., Day, R., Lamb, M. E., \& Cabrera, N. J. (2014). Should Researchers Conceptualize Differently the Dimensions of Parenting for Fathers and Mothers? Journal of Family Theory \& Review, 6(Dec), 390-405.

Fantinato, A. C. \& Cia, F. (2015). Habilidades Sociais Educativas, Relacionamento Conjugal e Comportamento Infantil na Visão Paterna: Um Estudo Correlacional. Psico, 46(1), 120-128. http://dx.doi.org/10.15448/1980-8623.2015.1.17330

Gracioli, S. M. A. \& Linhares, M. B. M. (2014). Temperamento e sua relação com problemas emocionais e de comportamento em pré-escolares. Psicologia em Estudo, 19(1), 71-80. http://dx.doi.org/10.1590/1413-7372189590007

Grusec, J. E. (2011). Socialization Processes in the Family: Social and Emotional Development. Annual Review of Psychology, 62, 243-269. http://dx.doi.org/10.1146/annurev.psych.121208.131650

Gryczkowski, M. R., Jordan, S. S., \& Mercer, S. H. (2010). Differential Relations between Mothers' and Fathers' Parenting Practices and Child Externalizing Behavior. Journal of Child and Family Studies, 19(5), 539-546. http:// dx.doi.org/10.1007/s10826-009-9326-2

Guilhardi, H. J. (2002). Auto-estima, autoconfiança e responsabilidade. In M. Z. S. Brandão \& F. C. S. Conte (Ed.), Comportamento Humano - Tudo (ou quase tudo) que você precisa saber para viver melhor. Santo André, SP: Esetec.

Harper, S. E. (2010). Exploring the Role of Filipino Fathers: Paternal Behaviors and Child Outcomes. Journal of Family Issues, 31(1), 66-89. http://dx.doi.org/10.1177/0192513X09342858

Jenkins, J. (2012). Understanding Multilevel Dynamics in the Development of Parenting. Parenting, 12(2-3), 254-260. http://dx.doi.org/10.1080/15295192.2012.683368

Lamb, M. E. (2012). The changing faces of fatherhood and father-child relationships: From fatherhood as status to father as dad. In M. A. Fine \& F. D. Fincham (Eds.), Handbook of Family Theories: A Content-Based Approach. Routledge.

Leme, V. B. R. \& Marturano, E. M. (2014). Preditores de comportamentos e competência acadêmica de crianças de famílias nucleares, monoparentais e recasadas. Psicologia: Reflexão e Crítica, 27(1), 153-162. http://dx.doi. org/10.1590/s0102-79722014000100017

Lins, T., Alvarenga, P., Paixão, C., Almeida, E., \& Costa, H. (2012). Problemas externalizantes e agressividade infantil: uma revisão de estudos brasileiros. Arquivos Brasileiros de Psicologia, 64(3). 
Marin, A. H., Piccinini, C. A., Gonçalves, T. R., \& Tudge, J. R. H. (2012). Práticas educativas parentais , problemas de comportamento e competência social de crianças em idade pré-escolar. Estudos de Psicologia, 17(1), 5-13. http:// dx.doi.org/10.1590/S1413-294X2012000100002

McLoyd, V. C., Cauce, A. M., Takeuchi, D., \& Wilson, L. (2000). Marital Processes and Parental Socialization in Families of Color: A Decade Review of Research. Journal of Marriage and the Family, 62(Nov), 1070-1093. http:// dx.doi.org/10.1111/j.1741-3737.2000.01070.x

Murray, K. W., Dwyer, K. M., Rubin, K. H., Knighton-Wisor, S., \& Booth-LaForce, C. (2014). Parent-Child Relationships, Parental Psychological Control, and Aggression: Maternal and Paternal Relationships. Journal of Youth and Adolescence, 43(8), 1361-1373. http://dx.doi.org/10.1007/s10964-013-0019-1

Patterson, G. R. (2002). Etiology and treatment of child and adolescent antisocial behavior. The Behavior Analyst Today, 3(2), 133-144. http://dx.doi.org/10.1037/h0099971

Patterson, G. R., Reid, J. B., \& Dishion, T. J. (1992). Antisocial Boys. Eugene: Castalia.

Phares, V., Lopez, E., Fields, S., Kamboukos, D., \& Duhig, A. M. (2005). Are fathers involved in pediatric psychology research and treatment? Journal of Pediatric Psychology, 30(8), 631-43. http://dx.doi.org/10.1093/jpepsy/jsi050

Pougnet, E., Serbin, L. A., Stack, D. M., \& Schwartzman, A. E. (2011). Fathers' influence on children's cognitive and behavioural functioning: A longitudinal study of canadian families. Canadian Journal of Behavioural Science, 43(3), 173-182. http://dx.doi.org/10.1037/a0023948

Rabinovich, E. P., Moreira, L. V. de C., \& Franco, A. (2012). Papéis, comportamentos, atividades e relações entre membros da família baiana. Psicologia \& Sociedade, 24(1), 139-149. http://dx.doi.org/10.1590/S0102-71822012000100016

Raya, A. F., Pino, M. J., \& Herruzo, J. (2011). Family variables related to behavioral problems in childhood. Israel Journal of Psychiatry, 48(2), 117-122.

Rohenkohl, L. M. I. A. \& Castro, E. K. (2012). Afetividade, conflito familiar e problemas de comportamento em pré-escolares de famílias de baixa renda: Visão de mães e professoras. Psicologia, Ciência e Profissão, 32(2), 438-451. http://dx.doi.org/10.1590/S1414-98932012000200012

Sarkadi, A., Kristiansson, R., Oberklaid, F., \& Bremberg, S. (2008). Fathers' involvement and children's developmental outcomes: a systematic review of longitudinal studies. Acta Paediatrica, 97(2), 153-158. http://dx.doi.org/10.1111/ j.1651-2227.2007.00572.x

Shaw, D. S., Bell, R. Q., \& Gilliom, M. (2000). A Truly Early Starter Model of Antisocial Behavior Revisited. Clinical Child and Family Psychology Rewiew, 3(3), 155-172. http://dx.doi.org/10.1023/A:1009599208790

Sherman, B. J., Duarte, C. S., \& Verdeli, H. (2011). Internalizing and Externalizing Problems in Adolescents from Bahia, Brazil. International Journal of Mental Health, 40(3), 55-76. http://dx.doi.org/10.2753/IMH0020-7411400304

Sidman, M. (2003). Coerção e suas implicações. Campinas: Livro Pleno (Originalmente publicado em 1989).

Todorov, J. C. (2001). Quem tem medo de punição? Revista Brasileira de Terapia Comportamental e Cognitiva, 3(1), $37-40$.

Torres, N., Verissimo, M., Monteiro, L., Ribeiro, O., \& Santos, A. J. (2014). Domains of father involvement, social competence and problem behavior in preschool children. Journal of Family Studies, 20(3), 188-203.

Trautmann-Villalba, P., Gschwendt, M., Schmidt, M. H., \& Laucht, M. (2006). Father-infant interaction patterns as precursors of children's later externalizing behavior problems: a longitudinal study over 11 years. European Archives of Psychiatry and Clinical Neuroscience, 256(6), 344-9. http://dx.doi.org/10.1007/s00406-006-0642-x

Verhoeven, M., Junger, M., van Aken, C., Deković, M., \& van Aken, M. A. G. (2010). Mothering, fathering, and externalizing behavior in toddler boys. Journal of Marriage and Family, 72(2), 307-317. http://dx.doi.org/10.1111/ j.1741-3737.2010.00701.x

Zhang, X. (2013). Bidirectional longitudinal relations between father - child relationships and Chinese children's social competence during early childhood. Early Childhood Research Quarterly, 28, 83-93. http://dx.doi.org/10.1016/j. ecresq.2012.06.005

Autores:

Luna Maiana Araújo Freitas - Mestre, Universidade Federal da Bahia (UFBA).

Patrícia Alvarenga - Doutora, Universidade Federal da Bahia (UFBA).

Endereço para correspondência:

Luna Maiana Araújo Freitas

Rua Aimoré, 278 - Parque Ipê

Feira de Santana, BA, Brasil

<lunamaiana@hotmail.com>

Recebido em: 22.02.2016

Aceito em: 24.08.2016 